\title{
Fast Large Volume Simulations of the Epoch of Reionization
}

\author{
M. G. Santos`, L. Ferramacho and M. B. Silva
}

CENTRA, Departamento de Física, Instituto Superior Técnico, 1049-001 Lisboa, Portugal

\begin{abstract}
We present a new type of simulation of the $21 \mathrm{~cm}$ signal from the Reionization and pre-Reionization epoch capable of quickly generating the signal up to very high redshifts with the large field of view of the next generation of radio telescopes. This simulation uses a semi-numerical prescription based on 3-d Monte-Carlo realizations of the dark matter density field, achieving a much higher dynamical range than previous time-consuming $\mathrm{N}$-body codes with radiative transfer algorithms. The simulation extends to high redshifts $(z \sim 25)$ thus including the effect of the Lyman alpha and collisional coupling as well as X-ray heating and corrections due to the gas bulk velocities. With the new algorithm we were able to achieve very large volumes $(1000 \mathrm{Mpc})^{3}$, thus fully probing the large scale structure of the $21 \mathrm{~cm}$ signal and making it ideal for the SKA simulated skies. This fast simulation also allows to quickly test the effect of different astrophysical parameters on the $21 \mathrm{~cm}$ signal and the code will be made publicly available for the benefit of the SKA community at large.
\end{abstract}

\section{Introduction}

Observations of the $21 \mathrm{~cm}$ line of neutral hydrogen are currently considered to be one of the most promising probes of the epoch of reionization (EoR) and possibly even the preceding period, during the so called dark ages. Motivated by the observational possibilities offered by the current and upcoming low frequency radio interferometers ${ }^{\mathrm{abc}}$ a great deal of effort has been underway in order to fully understand and generate the expected $21 \mathrm{~cm}$ signal that will be seen by these experiments (see Furlanetto et al. 2006 for a review).

Numerical simulations can potentially provide an improved description of the $21 \mathrm{~cm}$ brightness temperature signal and typically involve a combination of a N-body algorithm for dark matter, coupled to a prescription for baryons and star formation (often complemented by a hydrodynamical simulation), plus a full radiative transfer code to propagate the ionizing radiation (Gnedin 2000; Ciardi et al. 2003; Iliev et al. 2006; Zahn et al. 2007; Trac \& Cen 2007; Baek et al. 2009). These simulations have the advantage of calculating the signal from first principles, properly dealing with small scale details such as recombinations. The disadvantage is that they are slow to run, being constrained in dynamical range to sizes typically smaller than $100 \mathrm{Mpc} / \mathrm{h}$, and producing a large set of simulations for cosmological and astrophysical parameter estimates is unrealistic.

Another possibility is to use hybrid approaches involving simulations and analytical techniques (Thomas et al. 2009; Mesinger \& Furlanetto 2007). Although much faster than previous numerical methods, so far they have been constrained to "small" sizes depending on the amount of shared memory available in the computer and to low redshifts since it neglects the spin temperature evolution for the $21 \mathrm{~cm}$ calculation. Note that the proposed low-frequency interferometers that plan to observe the $21 \mathrm{~cm}$ signal from reionization have low spatial

^mgrsantos@ist.utl.pt

a LOFAR: http://lofar.org

b MWA: http://web.haystack.mit.edu/arrays/MWA

c SKA: http://www. skatelescope.org resolution capabilities but very large fields of view $\left(5 \times 5 \mathrm{deg}^{2}\right.$ or more), requiring boxes of at least $1000 \mathrm{Mpc}$ in size if we want to properly simulate a single field of view and pass such a simulation through the observation pipeline in order to understand how instrumental noise and systematic effects impact the observations. At the same time we need to be able to resolve halos of the order of $10^{8} M_{\odot}$ corresponding to a cooling temperature of $10^{4} \mathrm{~K}$ that is deemed necessary to host the ioniz ing sources responsible for reionization. This implies a resolution of $0.1 \mathrm{Mpc} / \mathrm{h}$ requiring the use of large boxes with $(7000)^{3}$ cells, which is beyond the capabilities of current hybrid methods. Moreover, at high redshifts we need to take into account detailed physics that determine the $21 \mathrm{~cm}$ brightness temperaf ture, such as the heating of the inter galactic medium (IGM) or the Lyman- $\alpha$ coupling of the spin temperature to the gas temperature, which makes it a challenging exercise to implement a complete radiative transfer/gas dynamics algorithm in a large volume simulation box.

We propose a semi-numerical technique, capable of quickly generating an end-to-end simulation of the $21 \mathrm{~cm}$ signal even at high redshifts when the spin temperature is non-negligible. Moreover, this method can be used to simulate very large volumes (e.g. $1000 \mathrm{Mpc}$ ), crucial to simulate the field-of-view of next generation of radio telescopes, without sacrificing the speed or requiring unpracticable amounts of computer memory. The code to generate this type of simulation will be provided publicly online ${ }^{\mathrm{d}}$ and it will be subject to continuous improvement through calibration against full radiative transfer/hydrodynamic simulations. The large volume simulations created with this method will be part of the SKA Design Studies Simulated Skies $\left(S^{3}\right)$ initiative and we hope this approach can prove useful to generate sky models for the future $21 \mathrm{~cm}$ experiments, which is crucial to test for calibration issues and foreground removal methods.

\footnotetext{
${ }^{d}$ http://www.simfast21.org
} 


\section{From linear density fluctuations to the ionization field}

The underlying basis for our simulation is the Monte-Carlo generation of the dark matter linear density field assuming a Gaussian probability distribution function for the linear overdensity as the initial state, which is then later evolved and used to find the collapsed structures and the corresponding ionized bubbles. This implementation partially follows the algorithm prescribed in Mesinger \& Furlanetto (2007), which is based on the "peak-patch" approach introduced by Bond \& Myers (1996). The principle behind this procedure is that at the high redshifts such as those where reionization occurs, the spatial distribution of dark matter halos can be obtained with linear theory, and non-linear gravitational effects can be accounted for by using only first-order perturbations on the linear field, i.e., with the Zel'dovich approximation (Zel'Dovich 1970; Efstathiou et al. 1985). For results presented here, we assumed a flat $\Lambda \mathrm{CDM}$ universe with the following cosmological parameters: $\Omega_{m}=0.28, \Omega_{b}=0.046, h=0.70, n_{s}=0.96$ and $\sigma_{8}=0.817$ based on the results from WMAP5, BAO and SN (see Hinshaw et al. 2009 and references therein).

The matter density fluctuations are first generated in Fourier space in a box with comoving size L and $\mathrm{N}$ cells, using Gaussian random variables with zero mean and unit variance set by $P_{\delta \delta}(k)$, the dark matter power spectrum. We define the power spectrum $P_{a a}(k)$ for a generic quantity $a$, as $\left\langle a(\mathbf{k}) a\left(\mathbf{k}^{\prime}\right)^{*}\right\rangle=(2 \pi)^{3} \delta_{D}^{3}\left(\mathbf{k}-\mathbf{k}^{\prime}\right) P_{a a}(k)$ for continuous fields, where $\delta_{D}$ is the Dirac delta function.

The density field is then transformed to real space using a Fast Fourier Transform (we used the fftw-3.1.1 package ${ }^{\mathrm{e}}$ ). This realization can be used to identify collapsed halos using the excursion-set formalism. The algorithm applies a series of top-hat filters of decreasing size to obtain the mean density around any given point of the density field, $\delta(M, x)$. Note that in order to make the process faster, we applied the filtering procedure as a multiplication in Fourier space (with the corresponding Fourier transform of the window function) and then transformed back to real space. A point is then considered to be the center of a halo of mass $M$ when the condition $\delta(M, x)>\delta_{c}(M, z)$ is verified, where $\delta_{c}(M, z)$ is the critical value from Sheth \& Tormen (1999).

We consider also a full exclusion criterion, which means that halos overlapping with a previously marked halo are discarded. Taking into account the available RAM memory and the above spatial resolution necessary to resolve the $10^{8} M_{\odot}$ halos $(0.1 \mathrm{Mpc} / \mathrm{h})$, we generated a simulation of comoving size $\mathrm{L}=300 \mathrm{Mpc}$ for $\mathrm{N}=1800^{3}$ cells. For the results presented here we used a machine with 32 CPUs and 64 GB of shared RAM memory, although only 20 CPUs were used at any given time.

Looking at figure 1 we can see that our simulations agree quite well with the theoretical curve and N-body simulation, although the number of low mass halos in the simulation with the lower resolution $(\mathrm{L}=300 \mathrm{Mpc}$ ) is slightly underestimated. However, we have checked that this effect has a minor impact on the ionized hydrogen distribution and can be seen as a good

\footnotetext{
e http://www.fftw.org
}

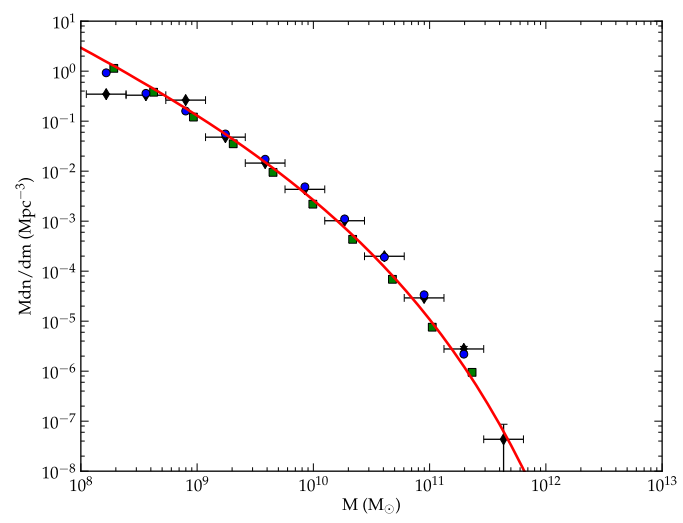

Fig. 1: Mass functions at $\mathrm{z}=10$ taken from our halo filtering prescrip tion with $\mathrm{L}=142 \mathrm{Mpc}$ and $\mathrm{N}=1536^{3}$ (blue circles) and $\mathrm{L}=300 \mathrm{Mpc}$ and $\mathrm{N}=1800^{3}$ (black diamonds). The results for the $\mathrm{N}$-body simulation of Trac et al. (2008) with $\mathrm{L}=142 \mathrm{Mpc}$ are also shown as green squares. The line show the mass function from Sheth \& Tormen (1999), using the fitting parameters by Jenkins et al. (2001). Error bars are included only for the $\mathrm{L}=300 \mathrm{Mpc}$ data for viewing purposes.

compromise in order to cover the largest possible volume with this algorithm.

With the halo distribution derived from the linear density field, the positions of both the halos and the dark matter were corrected to include non-linear dynamics. This was done by the means of the Zel'dovich approximation, i.e., by using the linear velocity field, obtained from our simulations, to adjust the positions of both the halos and dark matter distribution, starting at an arbitrary high redshift (see Mesinger \& Furlanetto 2007 for details on this implementation). To keep the maximum of information at all scales we decided to perform all corrections with $1800^{3}$ boxes and smooth the density and halo distributions boxes for speed purposes, only after applying the Zel'dovich corrections. With the corrected halo and density fields, the ionization regions can be determined using a similar excursionset algorithm. The principle behind this procedure is that the galaxies formed inside dark matter halos will produce a given amount of photons (dependent of the halo mass) that will ionize the surrounding hydrogen generating ionized bubbles. The efficiency of this process can be quantified by one parameter $\zeta$, so that a region will be ionized when:

$$
f_{\text {coll }} \geq \zeta^{-1},
$$

where $f_{\text {coll }}$ is the fraction of mass that has collapsed in halos in that region (Furlanetto et al. 2004).

We use this principle to construct our ionization field $\left(x_{i}\right)$, averaging both the halo mass and density fields with the spherical top-hat filters of decreasing size. Whenever the condition in equation (1) was verified, all cells contained inside the sphere where flagged as completely ionized, allowing at the same time for overlapping regions. We also considered the fractional description of the ionization state within each cell: after filtering our box up to the cell size, we searched for non ionized cells containing halos, to which we attributed an ionization value of $x_{i}=V_{i} / V_{c e l l}$, the ratio between the ionized volume which fol- 

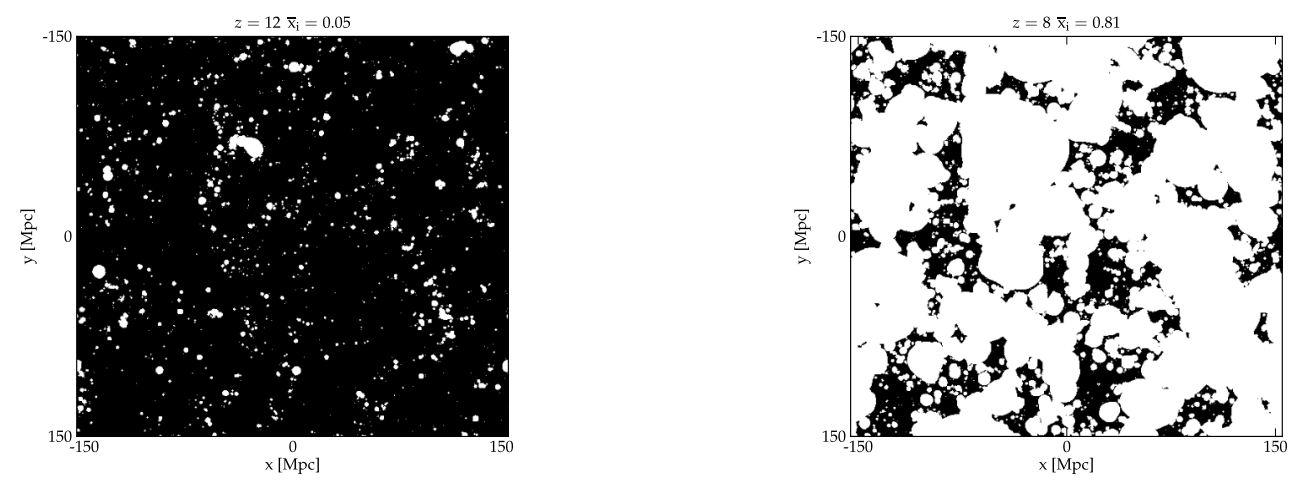

Fig. 2: Slices of the ionization field at the beginning and ending of reionization (left: $z=12, \bar{x}_{i}=0.05$; right: $z=8, \bar{x}_{i}=0.81$ )

lows directly from our barrier condition $V_{i}=M_{\text {coll }} \zeta /\left(1+\delta_{i}\right) \bar{\rho}_{m}$ and the cell volume.

As a result of this multi-step algorithm, we obtained the distribution of ionization bubbles in our simulation box which can be used to derive several relevant quantities. In figure 2 are shown two slices of our ionization field at the beginning and ending of reionization. These figures exhibit the characteristic bubble structure of ionized hydrogen increasing in size at lower redshifts until converging into a completely ionized IGM. Obviously, these evolution is intrinsically dependent on the assumed efficiency (here we used a value of 15.1), which could be constrained through observational data - by changing the efficiency parameter we can change the redshift where reionization starts and fit to a desired optical depth (we can quickly generate the ionization field once we change $\zeta$ ). Moreover this parameter can be easily changed in the code to a different model, such as a function dependent on redshift, without degradation of the speed of the simulation.

In order to characterize the statistical distribution of the ionization field we computed its power spectrum, $P_{x_{i} x_{i}}$ (figure 3 ). We see that the excursion-set formalism allows to obtain a bubble power spectrum that matches the one obtained from a full $\mathrm{N}$-body radiative transfer simulation when the volume size and cell resolution are the same. However, an important difference is observed between the power spectrum at large scales obtained in our simulation with a larger volume $(\mathrm{L}=300 \mathrm{Mpc})$ when compared to the lower volume ones. We believe this is due to the fact that we are finding larger bubbles in the 300 Mpc box, while smaller bubbles are absorbed in larger ones (a similar effect was observed in Mesinger \& Furlanetto 2007). We have checked that such differences do not originate from the lower number of low mass halos obtained in the $\mathrm{L}=300$ $\mathrm{Mpc}$ simulation or the fact that the mass function extends to larger masses in the larger simulation.

\section{Extending the simulation to very large volumes}

Although quite fast and based on first principle physics, the semi-numerical simulation presented so far still imposes some limitations regarding the volume it can cover. In the absence of large computing resources, it is not feasible to run a simulation covering a large dynamical range. For example the simulation presented above, covering $300^{3} \mathrm{Mpc}^{3}$, required to push to the limits of our machine using 20-processors and $64 \mathrm{~GB}$ of shared RAM in order to perform the FFT filtering. The main problem with the algorithm that was presented is that it still requires very large number of cells in order to achieve sufficient resolution to filter scales corresponding to $10^{8} \mathrm{M}_{\odot}$ halos and cover large volumes at the same time. If we want to simulate the very large volumes that will be surveyed by the next generation radio telescopes a less demanding algorithm is required.

This motivated us to implement a slightly simpler method when identifying the halos. Using the same resolution of $\mathrm{N}=1800^{3}$, but with $\mathrm{L}=1000 \mathrm{Mpc}$ (which corresponds to roughly a $5 \times 5 \mathrm{deg}^{2}$ sky at $\mathrm{z}=15$ ) the minimum cell size of $L / N \sim 0.36 \mathrm{Mpc} / \mathrm{h}$ corresponds to a minimum halo mass of $\sim 1 \times 10^{10} \mathrm{M}_{\odot}$. Clearly, this configuration does not allow us to resolve halos down to the required minimum mass of $10^{8}$ $\mathrm{M}_{\odot}$. However, one can use the expected halo mass function to place these smaller halos in our large volume simulation cells by using the prescription of Wilman et al. (2008). By the definition of the mass function, the quantity $n=d n / d M \Delta M \Delta V$ corresponds to the mean number of halos that can be found in a given cell with comoving volume $\Delta \mathrm{V}$. If one would simply perform a Poisson sampling for each cell with a mean $n$, the distribution of obtained halos would yield a correct mass function but their positions would be completely random. In order to correlate the halo positions with the underlying density field, a normalized bias term can be added,

$$
n_{c e l l}=K e^{b(z, M) \delta(z)} \frac{d n}{d M} \Delta M \delta V
$$

where $b(z, M)$ is the bias model for halos at a given redshift and $\delta(z, M)$ the density field at the same redshift. The factor $\mathrm{K}$ is a normalization constant that ensures the consistency of the above expression with the mass function $\frac{d n}{d M}$ when averaging over the large volume box. The above equation introduce a dependence of the halo locations on the density field, by amplifying the overdense regions with respect to underdense ones. As for the bias term, it describes the difference in clustering between dark matter halos and the mass density field. We consider a linear bias developed from the Press-Schechter formalism by 

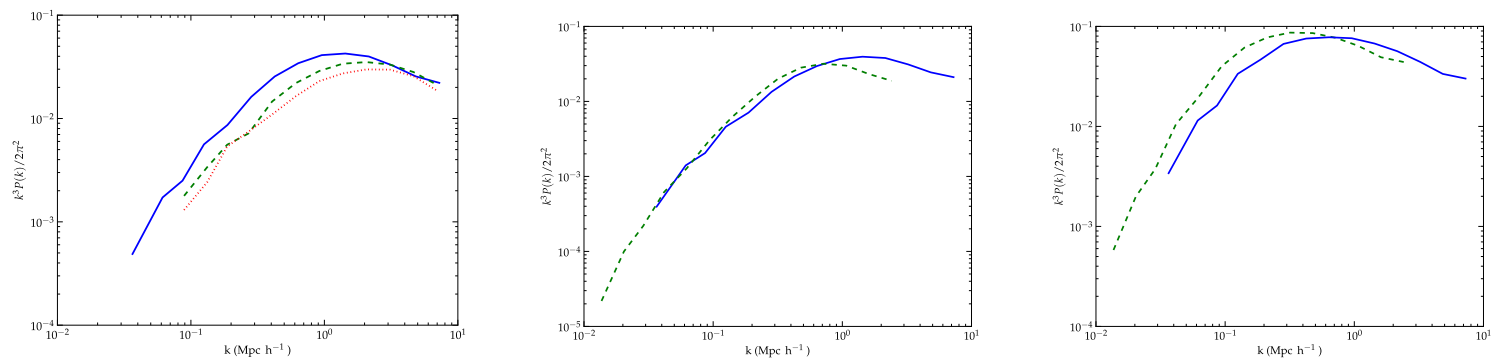

Fig. 3: First panel: power spectrum of the ionization field for our simulation with $\mathrm{L}=143 \mathrm{Mpc}, \mathrm{N}=768^{3}$ (red dotted curves), $\mathrm{L}=300 \mathrm{Mpc}, \mathrm{N}=600^{3}$ (solid blue curves) and the simulation of Trac et al. (2008) with $\mathrm{L}=143 \mathrm{Mpc}, \mathrm{N}=768^{3}$ (dashed green curves), for $z=9$ and $\bar{x}_{i}=0.15$. The boxes were smoothed to a common resolution. Right panels: power spectrum of the ionization field for the very large volume simulation (green dashed curves), compared to the same result for the $\mathrm{L}=300 \mathrm{Mpc}$ simulation. Both plots are for $\mathrm{z}=9$ but using different efficiency parameters $(\zeta=6.7$ and $\zeta=15.1)$ so that $\bar{x}_{i}=0.1$ (left) and $\bar{x}_{i}=0.55$ (right).

Mo \& White (1996) and later modified by Jing (1998):

$$
b(z, M)=\left(1+\frac{v-1}{\delta_{c}}\right)\left(\frac{1}{2 v^{4}-1}+1\right)^{0.06-0.02 n},
$$

where $v=\delta_{c} / \sigma(M)$ and $\mathrm{n}$ is the spectral index of the primordial density field fluctuation power spectrum.

We have then used this prescription in complement with the excursion-set formalism to perform a dark matter simulation with $\mathrm{L}=1000 \mathrm{Mpc}$. Since the cell size defines the lower mass scale using the exclusion set formalism, halos with mass above $10^{10} \mathrm{M}_{\odot}$ were obtained using this method. For scales bellow the cell size, we did a Poisson sampling of the entire box using eq. 2 for the distribution mean. In this way, we allow for the same cell to contain more than one halo, as long as the sum of their associated volumes does not exceed the cell volume $\Delta V$. In figure 4, we plot (blue diamonds) the obtained mass function for this run and compare it to the higher resolution, $\mathrm{L}=300$ Mpc simulation obtained using only the excursion set formalism. We can clearly see that only this method allows to have a high compatibility with the expected mass function with low resolution, whereas the full excursion-set formalism only has similar results when the cell resolution is considerably high. We also checked that this formalism can reproduce the spatial distribution of halos with its correlation with the underlying matter density field.

We then proceed to derive the ionization bubbles using the same method as in Section 2. The results for the power spectrum of the ionization field are shown in figure 3 (two right panels) compared to those obtained with the full excursion-set method with $\mathrm{L}=300 \mathrm{Mpc}$ and for two different efficiency parameters at $\mathrm{z}=9$. One observes that when the ionization fraction is rather low, the results are highly compatible due to the small bubble size in both cases. For an intermediate ionization state, the power at low scales is slightly larger in the $\mathrm{L}=1000 \mathrm{Mpc}$ indicating that by increasing the simulation volume the typical bubble size is also increased. This observation is consistent with what was observed using a pure excursion-set formalism, supporting our biased Poisson sampling algorithm for including low mass halos in large volume simulations.

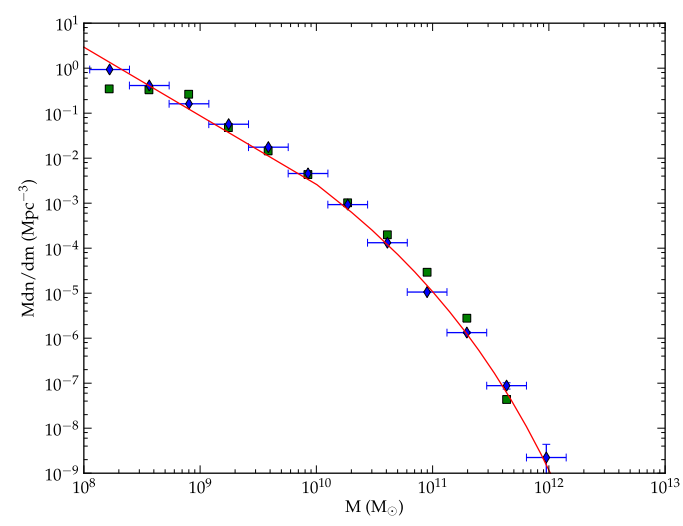

Fig. 4: Mass function at $\mathrm{z}=10$ for our simulation with $\mathrm{L}=300 \mathrm{Mpc}$ and $\mathrm{N}=1800^{3}$ (green squares) comparing with the large volume simulation with $\mathrm{L}=1000 \mathrm{Mpc}$ and $\mathrm{N}=1800^{3}$ (blue diamonds). Halos with masses $\mathrm{M}<10^{10} \mathrm{M}_{\odot}$ were placed using the Poisson sampling method introduced in this section.

\section{The $21 \mathrm{~cm}$ signal up to $z=25$}

With ionization maps at different redshifts we can focus on the predicted $21 \mathrm{~cm}$ signal from neutral hydrogen during the preEOR and the EOR. In the following Section we will briefly present the theoretical aspects behind this radiation and the results from our simulation. For a given observational frequency, the $21 \mathrm{~cm}$ signal corresponds to an intensity variation along the line of sight given by

$$
\begin{aligned}
& \delta T_{b}(v) \approx 23 f_{H I}(1+\delta)\left(1-\frac{T_{\gamma}}{T_{S}}\right)\left(\frac{h}{0.7}\right)^{-1}\left(\frac{\Omega_{b} h^{2}}{0.02}\right) \times \\
& {\left[\left(\frac{0.15}{\Omega_{m} h^{2}}\right)\left(\frac{1+z}{10}\right)\right]^{1 / 2}\left(\frac{1}{1+1 / H d v_{r} / d r}\right) \mathrm{mK},}
\end{aligned}
$$

where $T_{S}$ is the spin temperature of the IGM, $z$ is the redshift corresponding to the frequency of observation $\left(1+z=v_{21} / v\right)$, where $\left.v_{21}=1420 \mathrm{MHz}\right), T_{\gamma}=2.73(1+z) \mathrm{K}$ is the CMB temperature at redshift $z$ and $d v_{r} / d r$ is the comoving gradient of the line of sight component of the comoving velocity. In high density regions of the simulation one can have $1 / H d v_{r} / d r \leq-1$ 
and therefore we imposed a limit to this gradient of $d v_{r} / d r>$ $-0.7 \mathrm{H}$ in order to avoid singularities (this only affects ionized regions). In the above equation, both $f_{H I}$ and $\delta$ (as well as the velocity gradient) can be obtained as a direct result of our simulation (we used $f_{H I}=1-x_{i}$ ), but the term depending on $T_{S}$ is sensitive to variations of the spin temperature value over the volume. For $z<10$, one can make the reasonable assumption that $T_{s} \gg T_{\gamma}$ since the IGM gas temperature should be well above $100 \mathrm{~K}$ at these redshifts.

At high redshifts $(z \gtrsim 10)$ the spin temperature $\left(T_{S}\right)$ is no longer high enough to saturate the effect in equation 4 and we need to take into account the contribution of fluctuations from the spin temperature to the $21 \mathrm{~cm}$ signal. These originate from fluctuations in the coupling between the spin temperature and the gas temperature and the perturbations in the gas temperature itself $\left(T_{K}\right)$ and we can write:

$$
1-\frac{T_{\gamma}}{T_{S}}=\frac{x_{t o t}}{1+x_{t o t}}\left(1-\frac{T_{\gamma}}{T_{K}}\right),
$$

where $x_{t o t}=x_{\alpha}+x_{c}$ is the sum of the radiative and collisional coupling parameters. Collisions can be important for decoupling the HI $21 \mathrm{~cm}$ spin temperature from the CMB, especially at very high redshifts $(z \gtrsim 30)$ Nusser (2005) and are straightforward to apply to the simulation (Allison \& Dalgarno 1969; Zygelman 2005; Furlanetto \& Furlanetto 2007; Kuhlen et al. 2006; Hirata \& Sigurdson 2007). The radiative coupling due to the absorption of $L y_{\alpha}$ photons (the Wouthysen-Field effect, Wouthuysen 1952; Field 1959), on the other hand, should be dominant for $z<25$ and we shall concentrate on calculating this effect here. Note however that if we use a model where the $\mathrm{Ly}_{\alpha}$ coupling is smaller, then the collisional coupling at lower redshifts can become important close to the sources due to the X-ray emission (Zaroubi et al. 2007; Kuhlen et al. 2006). The radiative coupling is given by

$$
x_{\alpha}=\frac{S_{\alpha} J_{\alpha}}{J_{c}},
$$

with $J_{c} \approx 5.552 \times 10^{-8}(1+z) \mathrm{m}^{-2} \mathrm{~s}^{-1} \mathrm{~Hz}^{-1} \mathrm{sr}^{-1}$ and $S_{\alpha}$ is a correction factor of order unity (Chen \& Miralda-Escudé 2004; Hirata 2006; Chuzhoy \& Shapiro 2007; Furlanetto \& Pritchard 2006; Furlanetto et al. 2006). We follow the prescription in Santos et al. (2008) to calculate $J_{\alpha}$ (the spherical average of the number of Ly $\alpha$ photons hitting a gas element per unit proper area per unit time per unit frequency per steradian), given by a sum over the hydrogen levels $n$. For this, we need the comoving star formation rate, $\psi(\mathbf{x}, z)$ and $\epsilon_{\alpha}(v)$, the spectral distribution function of the sources (defined as the number of $L y_{n}$ photons per unit frequency emitted at $v$ per baryon in stars). We can easily assume any model for $\epsilon_{\alpha}(v)$ in our code to test for different sources of radiation. For this simulation we used: $A v^{-\alpha}$, with $\alpha=0.9$ and $A$ set such that we get a total of 20000 Ly $\alpha$ photons per baryon which gives a $L y_{n}$ emission similar to the one used in Santos et al. (2008). Note however that the amplitude is degenerate with the normalization of the star formation rate.

In order to obtain the star formation rate density from the simulation we used the halo catalogues generated to create boxes of collapsed mass $\left(M_{\text {coll }}(\mathbf{x}, z)\right)$ for two closely separated

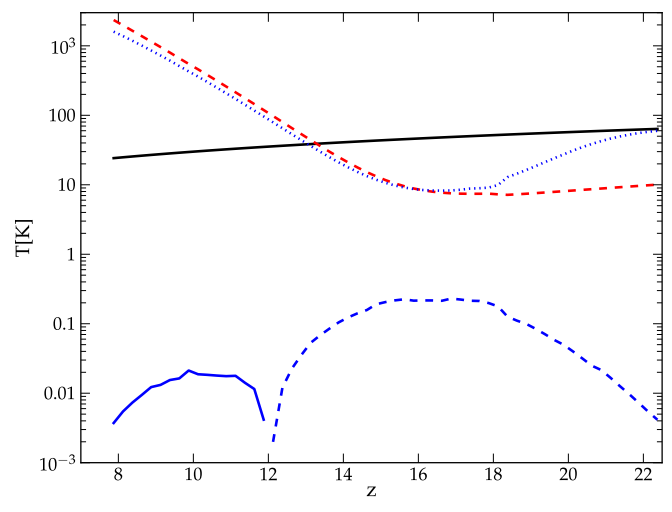

Fig. 5: The evolution of the gas temperature, spin temperature and brightness temperature with redshift. Solid black line - CMB; Red dashed - gas temperature; Blue dotted - spin temperature; Dark blue solid/dashed (below) - brightness temperature.

redshifts and calculated: $\psi(\mathbf{x}, z)=f_{*} \frac{d}{d t} M_{\text {coll }}(\mathbf{x}, z)$, where $f_{*}$ was used to normalize the star formation rate density to the one used in Shin et al. (2007). In the $J_{\alpha}$ calculation we can transform the integration over the volume into a convolution of the star for ${ }^{-} \Omega$ mation rate with a 1-D kernel, which can be quickly generated using a FFT.

The gas in the IGM is also heated as the reionization progresses. In our simulation, we assume that this heating is done by $\mathrm{x}$-rays with an emission connected to the star formation rate (see Santos et al. 2008). Our starting point was to calculate the total x-ray energy per unit time deposited in a given cell, for which we need the X-ray emissivity, $\epsilon_{X}(v)$, which is again assumed to be a power law, with a spectral index and amplitude compatible with what is observed for starburst galaxies (note that this amplitude is also degenerate with the star formation rate normalization). We then calculate how much of this energy is used for secondary ionizations in the neutral IGM, as well as how much is used to heat the IGM, taking into account the fraction of energy converted into ionizations and the fraction converted into heat (Shull \& van Steenberg 1985) which depends on the fraction of free electrons, $x_{e}$. We started the integration at $z=25$ assuming an initial temperature of $T=14 \mathrm{~K}$ consistent with adiabatic cooling of the gas from $z=150$ (where it is approximately equal to the $\mathrm{CMB}$ temperature). Figure 5, shows the evolution of the gas temperature with redshift, where we can see that most of the IGM is heated above $100 \mathrm{~K}$ for $z \lesssim 11$. The spin temperature starts very close to the CMB one (no signal) and approaches the gas temperature as $x_{\alpha}$ increases, following it for $z<17$.

Putting it all together we can calculate the $21 \mathrm{~cm}$ signal using equation 4. Calculation of $x_{\alpha}$ and the gas temperature takes around 5 minutes for each redshift in our machine using 20 CPUs. In figure 5, we can also see the evolution of the average brightness temperature with redshift, which is observed in absorption down to $z=12$ where it becomes approximately zero when $T_{K} \approx T_{C M B}$. In figure 6 we show maps of the $21 \mathrm{~cm}$ signal for a few very high redshifts taking into account all the ef- 

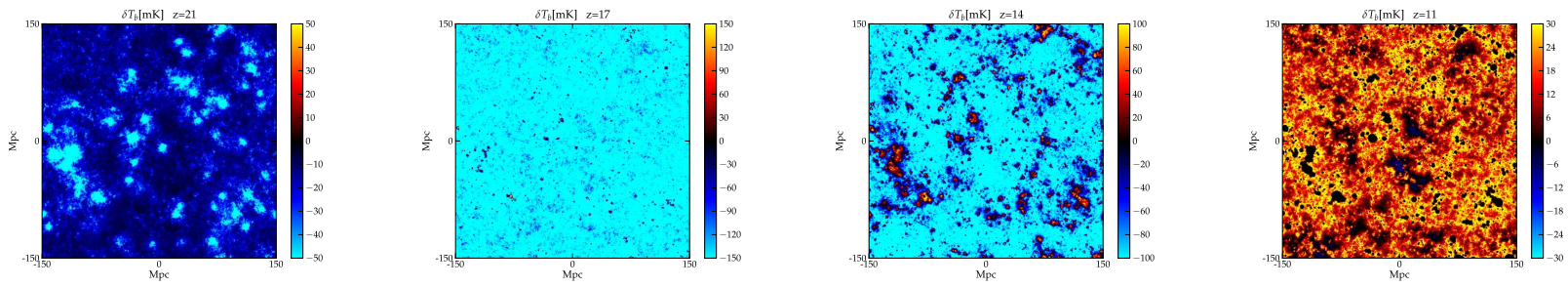

Fig. 6: Maps of the $21 \mathrm{~cm}$ signal at very high redshifts from the simulation, including all sources responsible for the fluctuations.

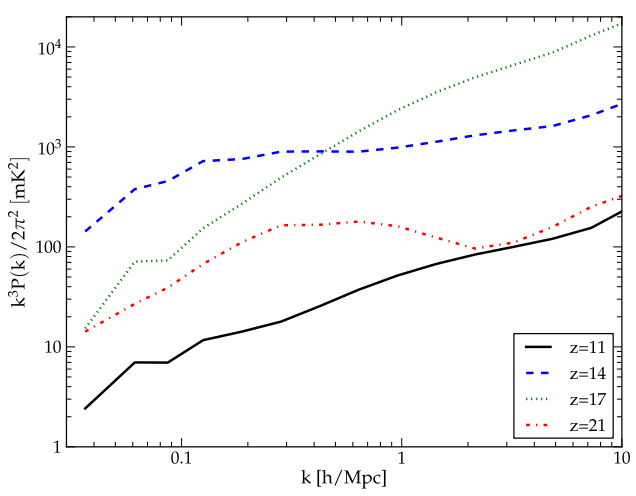

Fig. 7: The power spectrum of the $21-\mathrm{cm}$ signal at high redshifts including all fluctuations in the spin temperature.

fects. At high redshifts $(z=21)$ most of the temperature is zero $\left(T_{s} \sim T_{C M B}\right)$ but we already start to see some cold spots where the Ly $\alpha$ sources couple the spin temperature to the cold gas temperature and the fluctuations in $x_{\alpha}$ dominate. At $z=17$ the spin temperature is basically following the gas one everywhere and we can see fluctuations in the $21 \mathrm{~cm}$ signal due essentially to the gas temperature (although most of the Universe is still cold at this epoch). At $z=14$ we can already see the higher temperature regions surrounding the light sources located in the halos. Finally at $z=11$ basically all the IGM is heated above the $\mathrm{CMB}$ and we start seeing the ionization bubbles $\left(\bar{x}_{i} \approx 0.1\right.$, but note that some of the dark regions here are not due to the ionized bubbles but because $\left.T_{S}=T_{K} \sim T_{C M B}\right)$. Figure 7 shows the corresponding power spectrum. Note again that at $z \approx 11$ the fluctuations in the gas temperature still make an important contribution to the $21 \mathrm{~cm}$ signal, especially on large scales (increasing the power), even though the IGM temperature is already above the $\mathrm{CMB}$ one $\left(T_{K} \sim 150 \mathrm{~K}\right.$ and $\left.T_{C M B}=32.7 \mathrm{~K}\right)$.

Finally, we show the full brightness temperature map of the $21-\mathrm{cm}$ signal for a given redshift in the $\mathrm{L}=1000 \mathrm{Mpc}$ simulation (fig. 8), using the extension to larger volumes described in the previous section. This is an unprecedented large volume covered in a semi-numerical simulation of the EoR, capable of generating a $6 \times 6 \mathrm{deg}^{2}$ field of view.

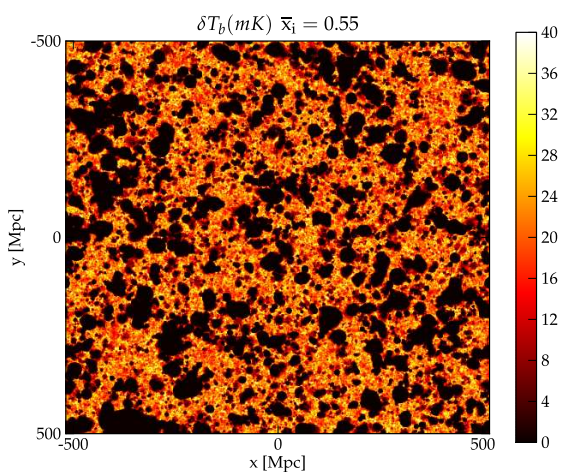

Fig. 8: The temperature map for the $\mathrm{L}=1000 \mathrm{Mpc}$ simulation at $\mathrm{z}=9$ with $\zeta=15.1$.

\section{Summary}

We presented a semi-numerical method capable of quickly generating end-to-end simulations of the $21 \mathrm{~cm}$ signal even at the high redshifts where the spin temperature is non-negligible (see Santos et al. 2009 for further details). The algorithm allows to generate brightness temperature boxes with very large volumes, e.g. $(1000 \mathrm{Mpc})^{3}$, crucial to properly simulate the field of view of the next generation of radio-telescopes, without sacrificing the speed or requiring unfeasible computer resources. The corresponding code (SimFast21) takes about 1 hour to run for each redshift, which can be considered remarkably fast. We are expecting that further optimization of the code can still reduce this time and also make it of the same order for higher values of the ionization fraction. This is on going research that will be implemented as we update the numerical code.

Although much faster than hydrodynamical numerical simulations, our analysis shows that relevant quantities such as the halo mass function, the halo mass power spectrum, the star formation rate or the ionization fraction power spectrum are all consistent with the numerical simulations with which we compared our results to. In particular, the ionization fraction power spectrum is similar to the one obtained with radiative transfer codes (RT) when comparing to the same volume. However, we still see an increase in the power spectrum at large scales when going to even larger volumes which are very hard to achieve with current RT methods. The difference is smaller as we look at smaller values of the ionization fraction, suggesting that this effect is due to the production of larger HII bubbles. 
The dependence on the astrophysical parameters of the simulation was encoded in three functions: the ionization efficiency, $\zeta$, the $\mathrm{Ly}_{\alpha}$ spectral distribution function of the sources, $\epsilon_{\alpha}$ and the X-ray spectral distribution function, $\epsilon_{X}$. These functions can be easily changed for a model of our choice and the code can then quickly generate new simulations of the signal (even faster if we keep the same cosmology). By combining all the unknowns into a physically meaningful small set of parameters we can easily probe the huge intrinsic parameter space available at high redshifts probed by $21 \mathrm{~cm}$ observations. This versatility is included in our algorithm. The possibility to generate reionization simulations from scratch without the need for supercomputers is a great advantage allowing to experiment with different models. Moreover, this allows room for improvement through calibration with full numerical simulations. The code can be a useful tool to generate sky models for future $21 \mathrm{~cm}$ experiments, important to test the observation pipeline, study how the foregrounds affect the observations and can be separated given a noise model, and to develop optimal estimators for signal extraction. The code will be released for public use in due course and we welcome the community participation in its updating and upgrading as well as development of additional applications on observational probes of reionization beyond the $21 \mathrm{~cm}$ observations.

Acknowledgements. We would like to thank R. Cen and H. Trac for providing data from their simulation. This work was partially supported by FCT-Portugal under grant PTDC/FIS/66825/2006. This work was supported by the European Commission Framework Program 6, Project SKADS, Square Kilometre Array Design Studies (SKADS), contract no 011938.

\section{References}

Allison, A. C. \& Dalgarno, A. 1969, ApJ, 158, 423

Baek, S., Di Matteo, P., Semelin, B., Combes, F., \& Revaz, Y. 2009, A\&A, 495, 389

Bond, J. R. \& Myers, S. T. 1996, ApJS, 103, 1

Chen, X. \& Miralda-Escudé, J. 2004, ApJ, 602, 1

Chuzhoy, L. \& Shapiro, P. R. 2007, ApJ, 655, 843

Ciardi, B., Ferrara, A., \& White, S. D. M. 2003, MNRAS, 344, L7

Efstathiou, G., Davis, M., White, S. D. M., \& Frenk, C. S. 1985, ApJS, 57, 241

Field, G. B. 1959, ApJ, 129, 536

Furlanetto, S. R. \& Furlanetto, M. R. 2007, MNRAS, 374, 547

Furlanetto, S. R., Oh, S. P., \& Briggs, F. H. 2006, Phys. Rep., 433, 181

Furlanetto, S. R. \& Pritchard, J. R. 2006, MNRAS, 372, 1093

Furlanetto, S. R., Zaldarriaga, M., \& Hernquist, L. 2004, ApJ, 613,1

Gnedin, N. Y. 2000, ApJ, 535, 530

Hinshaw, G., Weiland, J. L., Hill, R. S., et al. 2009, ApJS, 180, 225

Hirata, C. M. 2006, MNRAS, 367, 259

Hirata, C. M. \& Sigurdson, K. 2007, MNRAS, 375, 1241

Iliev, I. T., Mellema, G., Pen, U.-L., et al. 2006, MNRAS, 369, 1625
Jenkins, A., Frenk, C. S., White, S. D. M., et al. 2001, MNRAS, 321,372

Jing, Y. P. 1998, ApJ, 503, L9+

Kuhlen, M., Madau, P., \& Montgomery, R. 2006, ApJ, 637, L1

Mesinger, A. \& Furlanetto, S. 2007, ApJ, 669, 663

Mo, H. J. \& White, S. D. M. 1996, MNRAS, 282, 347

Nusser, A. 2005, MNRAS, 359, 183

Santos, M. G., Amblard, A., Pritchard, J., et al. 2008, ApJ, 689, 1

Santos, M. G., Ferramacho, L., Silva, M. B., Amblard, A., \& Cooray, A. 2009, ArXiv e-prints

Sheth, R. K. \& Tormen, G. 1999, MNRAS, 308, 119

Shin, M.-S., Trac, H., \& Cen, R. 2007, ArXiv e-prints, 708

Shull, J. M. \& van Steenberg, M. E. 1985, ApJ, 298, 268

Thomas, R. M., Zaroubi, S., Ciardi, B., et al. 2009, MNRAS, 393, 32

Trac, H. \& Cen, R. 2007, ApJ, 671, 1

Trac, H., Cen, R., \& Loeb, A. 2008, ApJ, 689, L81

Wilman, R. J., Miller, L., Jarvis, M. J., et al. 2008, MNRAS, 388,1335

Wouthuysen, S. A. 1952, AJ, 57, 31

Zahn, O., Lidz, A., McQuinn, M., et al. 2007, ApJ, 654, 12

Zaroubi, S., Thomas, R. M., Sugiyama, N., \& Silk, J. 2007 MNRAS, 375, 1269

Zel'Dovich, Y. B. 1970, A\&A, 5, 84

Zygelman, B. 2005, ApJ, 622, 1356 